\title{
DLC2/StarD13 plays a role of a tumor suppressor in astrocytoma
}

\author{
SALLY EL-SITT ${ }^{1}$, BASSEM D. KHALIL ${ }^{1}$, SAMER HANNA ${ }^{1}$, MARWAN EL-SABBAN ${ }^{2}$, \\ NAJLA FAKHREDDINE ${ }^{3}$ and MIRVAT EL-SIBAI ${ }^{1}$ \\ ${ }^{1}$ Department of Natural Sciences, The Lebanese American University, Beirut 1102 2801; ${ }^{2}$ Department of Anatomy, \\ Cell Biology and Physiological Sciences, Faculty of Medicine, The American University of Beirut, \\ Beirut; ${ }^{3}$ Department of Pathology, Hammoud Hospital, Saida, Lebanon
}

Received January 17, 2012; Accepted March 8, 2012

DOI: $10.3892 / o r .2012 .1819$

\begin{abstract}
Astrocytomas are tumors occurring in young adulthood. Astrocytic tumors can be classified into four grades according to histologic features: grades I, II, III and grade IV. Malignant tumors, those of grades III and IV, are characterized by uncontrolled proliferation, which is known to be regulated by the family of Rho GTPases. StarD13, a GAP for Rho GTPases, has been described as a tumor suppressor in hepatocellular carcinoma. In the present study, IHC analysis on grades I-IV brain tissues from patients showed StarD13 to be overexpressed in grades III and IV astrocytoma tumors when compared to grades I and II. However, when we mined the REMBRANDT data, we found that the mRNA levels of StarD13 are indeed higher in the higher grades but much lower than the normal tissues. Knocking down StarD13 using siRNA led to a decrease in cell death and an increase in cell viability, proving that StarD13 is indeed a tumor suppressor in astrocytomas. This was found to be mainly through cell cycle arrest independently of apoptosis. Finally, we detected an increase in p-ERK in StarD13 knockdown cells, uncovering a potential link between Rho GTPases and ERK activation.
\end{abstract}

\section{Introduction}

Primary malignant central nervous system (CNS) tumors account for $2 \%$ of all cancers with an inconsistent rate of morbidity and mortality. Malignant CNS tumors constitute the leading cause of death from solid tumors in children and the third leading cause of cancer-related death for adolescents and adults aged 15-34 years (1).

Astrocytomas are tumors occurring in young adulthood defined as CNS neoplasms originated in astrocytes, star-shaped brain cells $(2,3)$. The World Health Organization (WHO)

Correspondence to: Dr Mirvat El-Sibai, Department of Natural Sciences, Lebanese American University, P.O. Box 13-5053, Chouran Beirut 1102 2801, Lebanon

E-mail: mirvat.elsibai@lau.edu.lb

Key words: astrocytoma, Rho GTPases, StarD13, GAP, tumor suppressor system classifies the astrocytic tumors into four grades: grade I (pilocytic astrocytoma), grade II (diffuse astrocytoma) with cytological atypia alone, grade III (anaplastic astrocytoma) showing anaplasia and mitotic activity in addition and grade IV (glioblastoma) presenting microvascular proliferation and/ or necrosis (4). Malignant gliomas are those of grades III and IV (5). Glioblastomas account for at least $80 \%$ of malignant gliomas (6).

Rho GTPases are known to be involved in the stimulation of cell cycle progression. The family of Rho GTPases contains 20 small $\mathrm{G}$ proteins playing important roles in the regulation of the cytoskeleton, the cell cycle, the cell migration and the cell polarity (7). Rho GTPases are guanine nucleotide binding proteins existing in two forms: the active form which is GTP bound and the inactive one that being GDP bound and it is important to note that only in the active form, Rho GTPases can interact with other effectors mediating their cellular functions (8). The most important studied members of the Rho family are Rac 1, Cdc42 and Rho A (8).

Genetic screens of many human cancers have revealed altered expression of various Rho family GTPases (9). RhoC mRNA level is increased in metastatic melanomas and Rac3 activity is increased in highly proliferative breast cancer cell lines (9). Regulators of Rho GTPases also show aberrant expression in human tumors, including Vav1 in neuroblastomas (10). A comparison of the gene expression pattern in a metastatic breast cancer cell line compared to its non-metastatic counterpart revealed that many genes encoding actin regulatory proteins are more highly expressed in metastatic cells (11). All of these processes are regulated by the proteins of the Rho GTPase family making them with their regulators and effectors important in controlling tumor formation and progression in humans (7).

The regulation of Rho GTPases is governed by three classes of regulatory proteins: GAP (GTPase activating proteins), GEF (guanine nucleotide exchange factors), which accelerate the very slow intrinsic guanine nucleotide exchange and GTP hydrolysis activity, and GDI (guanine nucleotide dissociation inhibitors) $(12,13)$. GAPs belong to a specific family of GTPases that accelerate the rate of GTP hydrolysis by up to $10^{5}$ times (14). Hence, a tumor suppressor role has been suggested for GAPs counteracting the oncogenic potential of Rho proteins (15). Since the identification of the first RhoGAP (16) more than 50 RhoGAPs in the human genome were character- 
ized, three of which contain START domain: DLC1, DLC2 known also as START-GAP2 or StarD13 and DLC3 (17). The three DLC proteins are characterized by the presence of three motifs: a sterile $\alpha$ motif (SAM), a RhoGAP catalytic domain, and a START (Star-related lipid transfer) domain (18). The SAM domain consists of approximately 70 residues and has been shown to play several roles particularly as protein interaction modules because of its ability to interact with other SAM domains (19). SAM domains are located on the N-terminus and may bind to DNA or RNA (20). DLC2-SAM shows only 15-30\% homology with other SAM domains and is considered as the prototype in the family of the DLC2-related proteins (19).

When searching for additional candidate tumor suppressor loci critical in hepatocellular carcinoma after the well-known and established tumor suppressor genes $p 53, c-m y c, p 16^{\text {ink } 4}$ and $\beta$-catenin, Ching et al identified a novel gene $D L C 2$ on chromosome $13 q 12$ which was found to be underexpressed in hepatocellular carcinoma $(21,22)$. DLC2 which is also known as steroidogenic acute regulatory protein-related lipid transfer (START) domain containing protein 13 (StarD13), display high level of homology with DLC1 (deleted in liver cancer 1), a gene coding for a Rho GTPase activating protein. These two proteins have $51 \%$ identity and $64 \%$ similarity at the level of their amino acid sequences sharing the same SAM-RhoGAPSTART domain organization $(22,23)$. DLC1 is down-regulated in many types of cancers including lung, breast, prostate, kidney, colon, uterus, ovary, and stomach due to two major causes: genomic deletion and promoter hypermethylation.

Similar to DLC1, DLC2 is down-regulated in several types of cancer including lung, ovarian, renal, breast, uterine, gastric, colon and rectal tumors (23). Through its RhoGAP activity, the DLC2 protein acts on RhoA-C and Cdc42 but not on Rac1 $(13,21,24)$. Xiaorong et al reported significant correlations between underexpression of DLC2 and cell differentiation. In addition, a negative correlation was established between DLC2 and RhoA. DLC2 seemed to inhibit hepatocarcinogenesis by suppressing RhoA activity (25). On the other hand, a study conducted by Yau et al investigated the role of DLC2 by generating DLC2-deficient mice. The mice that were defective in DLC2 were able to survive to adulthood unlike the knockout of DLC1 which led to embryonic lethality (15).

In this study, we examine the role of StarD13 as a potential tumor suppressor in astrocytoma to compare it with the newly described role of StarD13 as a tumor suppressor for hepatocellular carcinoma.

\section{Materials and methods}

Cell culture. The human astrocytoma cell line T98G was cultured in DMEM medium supplemented with $10 \%$ FBS and $100 \mathrm{U}$ penicillin/streptomycin at $37^{\circ} \mathrm{C}$ and $5 \% \mathrm{CO}_{2}$ in a humidified chamber.

Antibodies and reagents. Goat polyclonal anti-StarD13 antibody was obtained from Santa Cruz Biotechnology. Mouse monoclonal anti-Rho, mouse monoclonal anti-Racl antibodies were purchased from Upstate Biotechnology (Lake Placid, NY). The anti-Cdc42 antibody (Sc-87) was obtained from Santa Cruz Biotechnology. Anti-goat and anti-mouse HRP-conjugated secondary antibodies were obtained from Promega. Fluorescent secondary antibodies (AlexaFluor 488) and rhodamin phalloidin were obtained from Invitrogen.

The full length GFP-StarD13 construct was a generous gift from Dr Hitoshi Yagisawa from the University of Hyogo, Japan [mammalian expression plasmids for GFP fusion proteins, pEGFPSTART-GAP1(wt)] (13).

Cell transfection and small interfering RNA. Cells were transfected with $5 \mu \mathrm{g}$ GFP-StarD13 or control empty GFP alone vectors using Lipfectamine LTX with Plus reagent (Invitrogen) as described by the manufacturer. Goat FlexiTube siRNA for StarD13 was obtained from Qiagen. The siRNAs used had the following target sequence: StarD13: 5'-CCCGCAATACGCTC AGTTATA-3'. The cells were transfected with the siRNA at a final concentration of $10 \mathrm{nM}$ using HiPerfect (Qiagen) as described by the manufacturer. Control cells were transfected with siRNA sequences targeting GL2 luciferase (Qiagen). After $72 \mathrm{~h}$, protein levels in total cell lysates were analyzed by western blotting using the appropriate antibodies or the effect of the corresponding knockdown was assayed.

Pull down assays and western blot analyses. For experiments, cells were starved in low-glucose Opti-MEM medium without FBS for $3 \mathrm{~h}$, and stimulated with a final concentration of $10 \mathrm{nM}$ recombinant human epidermal growth factor (Hu EGF) for various times (Invitrogen). Cells were then lysed and the pulldown assay performed using the RhoA/Rac1/Cdc42 Activation Assay Combo kit (Cell BioLabs) following the manufacturer's instructions. Briefly, cell lysates were incubated with GST-RBD (for Rho pull down) or GST-CRIB (for the Rac and Cdc42 pull down) for $1 \mathrm{~h}$ at $4^{\circ} \mathrm{C}$ with gentle agitation. Then, the samples were centrifuged, and the pellet washed several times. After the last wash, the pellets were resuspended with sample buffer and boiled for $5 \mathrm{~min}$. GTP-RhoA or GST-Rac/Cdc42 were detected by western blotting using anti-RhoA, anti-Rac or anti-Cdc42, respectively. Total RhoA/Rac and Cdc42 were collected prior to the incubation with GST-RBD/GST-CRIB and used as a loading control.

Immunohistochemistry. Permission for tissue collection was granted by the Committee on Human Subjects in Research (CHSR) at the Lebanese American University (approval given on March 26 2010, CHSR tracking no. NSMS26032010-1). Tissues were collected from Rizk Medical Center with the help of Dr Noha Bejjani and from Hammoud Hospital with the help of Dr Najla Fakhreddine. Frozen human astrocytoma tissues of grades I and IV were sectioned to $8 \mu \mathrm{m}$ sections using a refrigerated microtome. Tissues were fixed with $4 \%$ paraformaldehyde for $10 \mathrm{~min}$, and permeabilized with $0.5 \%$ Triton X-100 for $10 \mathrm{~min}$. To decrease background fluorescence, tissues were rinsed with $0.1 \mathrm{M}$ glycine then incubated with $0.1 \mathrm{M}$ glycine for $10 \mathrm{~min}$. For blocking, tissues were incubated 4 times with $1 \%$ BSA, $1 \%$ FBS in PBS for $5 \mathrm{~min}$. Samples were stained with the StarD13 primary antibody for $2 \mathrm{~h}$ and with a fluorophore-conjugated secondary antibody for $2 \mathrm{~h}$. Tissue fluorescent images were taken using a 10x objective on a Zeiss LSM confocal microscope. For image analysis, all digital images were imported in ImageJ software (National Institutes of Health, MA). The total fluorescence intensity of a fixed area from at least 10 different frames from each tissue was determined. 
Proliferation. Depending on the type of experiment, cells were seeded either in 12-well plate or in 96-well plate. After $24 \mathrm{~h}$ of seeding, cells were transfected with StarD13 siRNA or overexpressed with GFP-StarD13. At the end of each treatment period, cell viability was determined using the trypan blue exclusion method or the cell proliferation reagent (WST-1; Roche, Mannheim, Germany) as recommended by the manufacturer. Briefly, at the end of the treatment period, water-soluble tetrazolim salt (WST-1 was added to the cells and kept in a humidified incubator $\left(37^{\circ} \mathrm{C}\right)$ at $95 \%$ air and $5 \% \mathrm{CO}_{2}$ for $4 \mathrm{~h}$. The formazan dye was then measured colorimetrically at $450 \mathrm{~nm}$. The results were expressed as percent of control.

Flow cytometry. Treated cells were placed into $15 \mathrm{ml}$ Falcon tubes and centrifuged at $1500 \mathrm{rpm}$ for $5 \mathrm{~min}$. The pellet was then washed by resuspending it in $1 \mathrm{ml}$ of ice-cold $1 \mathrm{X}$ phosphate buffered saline (PBS) followed by $4 \mathrm{ml}$ of $70 \%$ ethanol. Cells were then treated with $100 \mu \mathrm{l}$ of RNase and incubated for $1 \mathrm{~h}$ at $37^{\circ} \mathrm{C}$. The cells were then pelleted at $2000 \mathrm{rpm}$ for $5 \mathrm{~min}$, and the pellets were washed with $500 \mu 1$ of $1 \mathrm{X}$ PBS, transferred to labeled $6 \mathrm{ml}$ polystyrene round bottom falcon tube, and stained with $30 \mu \mathrm{l}$ propidium iodide for $10 \mathrm{~min}$ in the dark. Cells were analyzed using a FACScan and the DNA content determined by CellQuest software.

Annexin staining. Cells were trypsinized and centrifuged at $1500 \mathrm{rpm}$ for $5 \mathrm{~min}$. The pellet was then washed by resuspending it in $1 \mathrm{ml}$ of ice-phosphate buffered saline (PBS). Cells were centrifuged again under the same conditions as before. Cells were then stained with $5 \mu \mathrm{l}$ of Annexin V-FITC and $10 \mu \mathrm{l}$ of propidium iodide cells and incubated at room temperature for $10 \mathrm{~min}$ and protected from light. The fluorescence of the cells was determined immediately with a flow cytometer.

REMBRANDT database. To determine the expression of StarD13 in human gliomas, we mined the publicly available Repository for Molecular Brain Neoplasia Data (REMBRANDT) gene expression microarray database containing 452 clinically annotated brain tumor specimens (National Cancer Institute, 2005; REMBRANDT home page: <http://rembrandt.nci.nih. gov>; accessed December 20, 2010). We specifically examined the gene expression data from nonneoplastic brain $(\mathrm{NB}, \mathrm{n}=28)$, low-grade astrocytomas (LGGs, $n=148$ ), and glioblastoma multiformes (GBMs, $\mathrm{n}=226)$.

\section{Results}

StarD13 is a GAP for RhoA and Cdc42 not for Racl. In order to study the role of StarD13 in astrocytoma malignancy, we started by verifying that StarD13 is a GAP for RhoA and Cdc42 and not for Rac1. This was achieved by studying the activation of the three Rho GTPases in T98 cells following the transfection by GFP-StarD13. Using a GST-RBD (Rho binding domain from Rhotekin) and GST-CRIB pull down assay, we looked for GTP-loaded Rho and Cdc42/Rac, respectively. We found that the levels of the active Rho and Cdc 42 were lower in cells transfected with GFP-StarD13 (Fig. 1A and B) as compared to the controls. On the other hand, the overexpression of StarD13 did not affect the active Rac1 (Fig. 1C). This confirmed that StarD13 is a specific GAP for Rho and Cdc42.

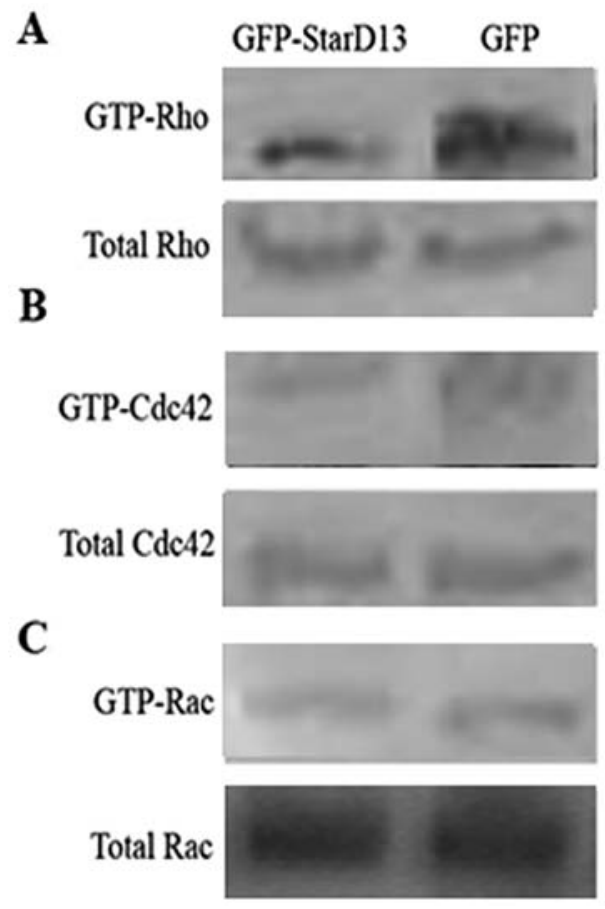

Figure 1. StardD13 is a specific GAP for Rho and Cdc42. T98G cells were transfected with either GFP alone (right lanes) or with GFP-StarD13 (left lanes). The cells were then lyzed and incubated with (A) GST-RBD (Rhotekin binding domain), or (B) GST-CRIB (Cdc42 and Rac interactive binding domain) to pull down active Rho or $\mathrm{Cdc} 42$ and Rac, respectively. The samples were then blotted with Rho, Cdc42 and Rac antibodies. The lower gels in each panel are western blot analyses for the total cell lysates for loading control.

StarD13 is underexpressed in astrocytoma. Then we investigated the expression levels of StarD13 in astrocy toma malignancy using immunohistochemistry. Tissues of grades I and IV were stained with anti-StarD13 antibody (Fig. 2A) and the intensity of the signal was measured using ImageJ software. Our results showed that there was $\sim 30 \%$ increase in the expression of StarD13 in grade IV tumors as compared to grade I (Fig. 2B).

StarD13 overexpression reduces cell viability. In order to determine the role of StarD13 in cell viability, we transfected T98 cells with GFP-StarD13 and studied the effect of this overexpression on cell viability using two methods: the trypan blue exclusion method and the cell proliferation reagent (WST-1).

The overexpression was apparent looking at the GFP channel and through the effect of overexpressing GFP-StaD13 on stress fiber formation (due to Rho inhibition) as revealed by rhodamine phalloidin staining (Fig. 3D). This is compared to cells transfected with GFP alone (Fig. 3D, upper panels) where stress fibers were not affected. The overexpression of StarD13 decreased the percentage of live cells from 97 to $92 \%$ (Fig. 3A). This was consistent with the results of the WST-1 which showed a decrease of $20 \%$ in cell viability (Fig. 3C). The amount of dead cells increased by $\sim 2$-fold (Fig. 3B).

StarD13 knockdown increases cell viability. To confirm the previous results, we knocked down expression of StarD13 with siRNA. StarD13 expression was reduced by $50 \%$ as compared to cells transfected with control siRNA duplexes (Fig. 4D). The percentage of live cells in StarD13-siRNA treated cells was 
A

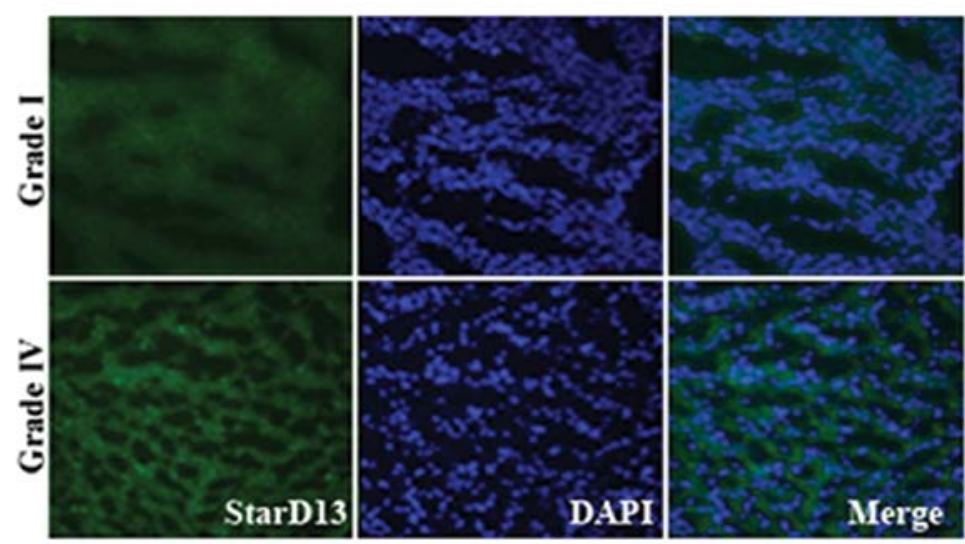

B

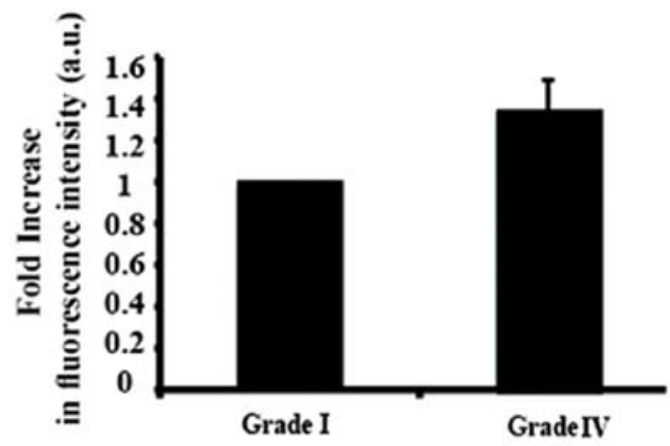

Figure 2. StarD13 is overexpressed in grade IV astrocytoma compared to grade I. Immunohistochemistry for tumor tissues of grades I and IV that were obtained from patients diagnosed with glioblastoma. (A) Representative micrographs of tissues that were stained with DAPI (middle panels) or with anti-StarD13 (left panels). (B) The intensity of the signal was measured using ImageJ software and expressed as fold increase to grade I. Data are the mean \pm SEM.

$\mathbf{A}$

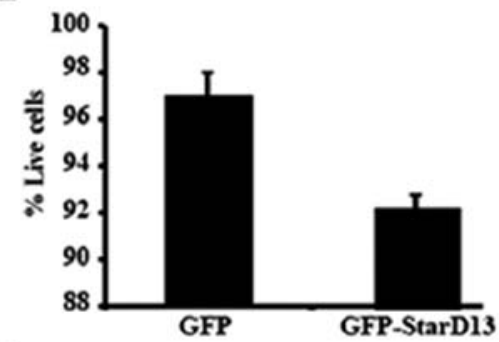

C

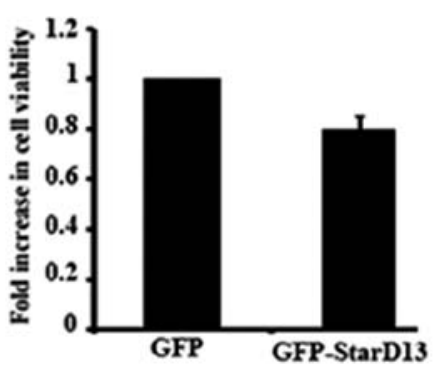

B

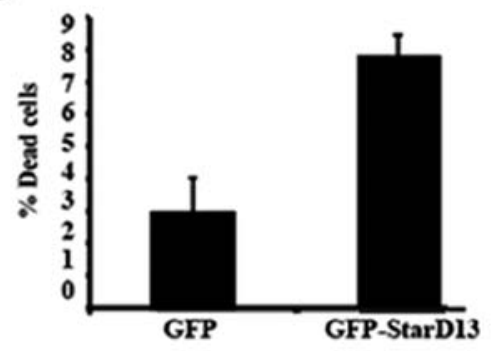

D

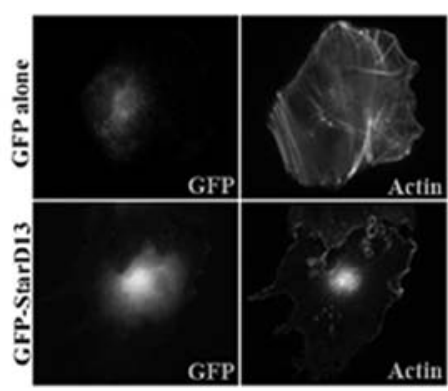

Figure 3. StarD13 overexpression decreased cell viability. Cells were either transfected with GFP alone or with GFP-StarD13. Cell viability was then determined using the trypan blue exclusion method (A and B) or the WST-1 cell proliferation reagent (C). (A and B) The results were expressed as percent of total number of cells. C) Results were expressed as fold increase from control (GFP alone). Data are the mean \pm SEM. (D) Cell were transfected with GFP alone (upper panel) or GFP-StarD13 (lower panel) and stained with rhodamine phalloidin.

increased as compared to control-siRNA treated cells (Fig. 4A). These results were consistent with those of the WST-1 which showed an increase of about $30 \%$ in cell viability (Fig. 4C). The amount of dead cells increased by $\sim 2$-fold (Fig. 4B).
StarD13 is overexpressed in glioblastoma. Surprisingly, our immunochemistry results suggested StarD13 might be an oncogene and not a tumor suppressor, as the literature suggested, since StarD13 looked to be overexpressed in grade IV astro- 


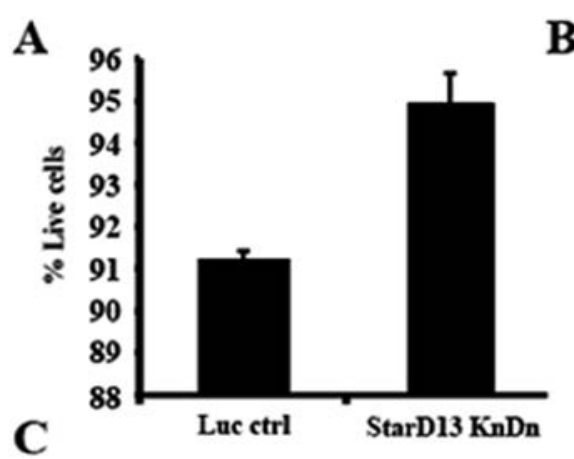

B
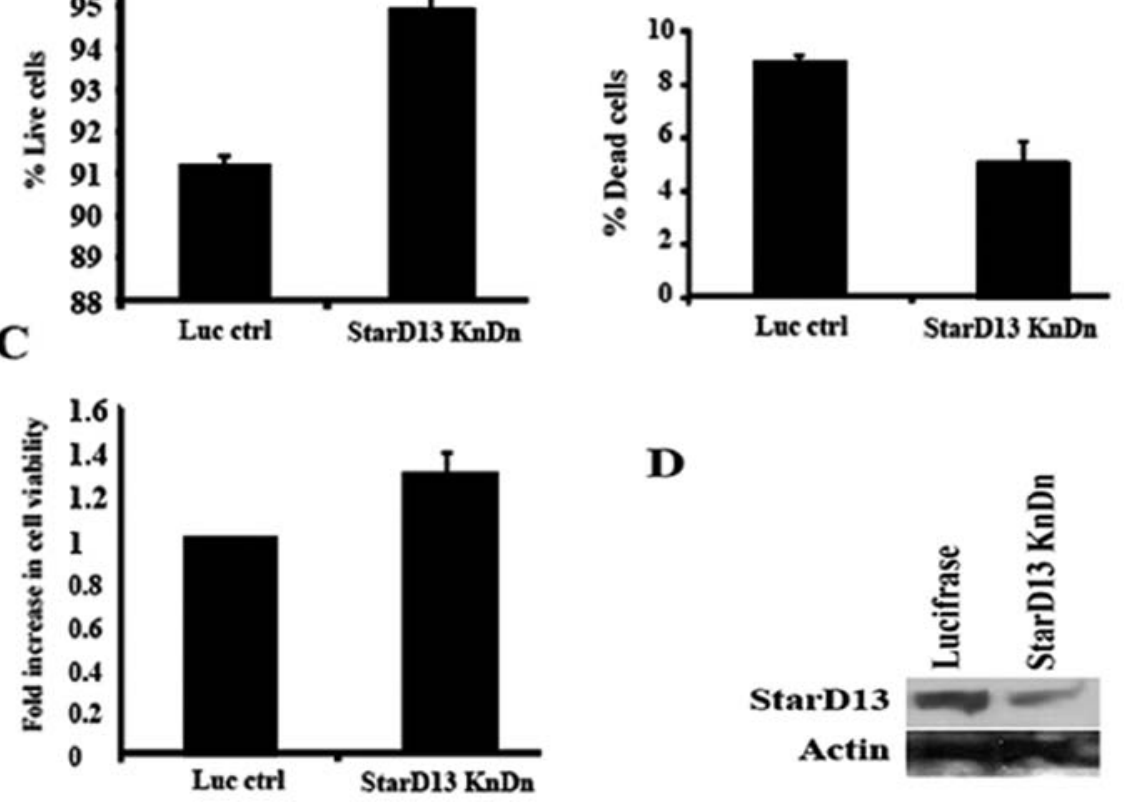

D

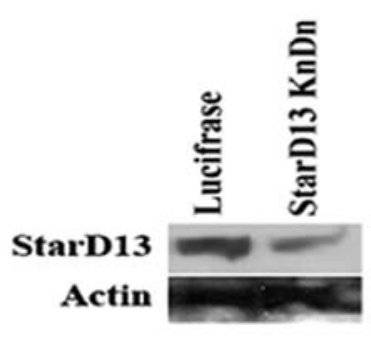

Figure 4. StarD13 underexpression increases cell viability. Cells were either transfected with luciferase control siRNA or with StarD13 siRNA. Cell viability was then determined using the trypan blue exclusion method (A) and (B) or the WST-1 cell proliferation reagent (C). (A and B) The results were expressed as percent of total number of cells. (C) Results were expressed as fold increase from control (luciferase). Data are the mean \pm SEM. (D) Western blot analysis showing the decrease in StarD13 expression compared to actin (lower panel), in cells transfected with StarD13 siRNA compared to luciferase control (left lane).
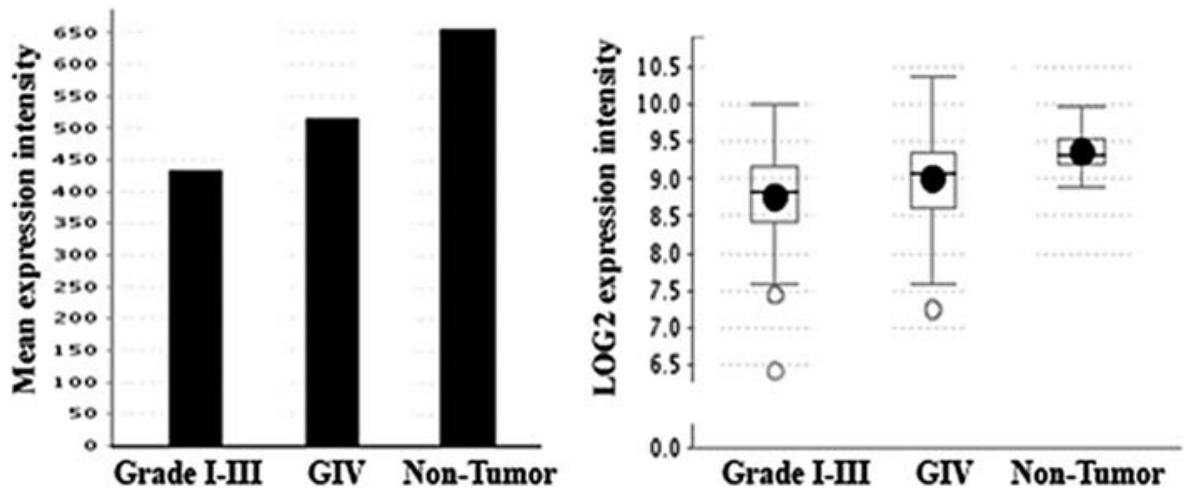

Figure 5. StarD13 is underexpressed in glioma as compared to normal tissues. Data analyzed from REMBRANDT website. mRNA from 28 non-tumor patient tissues, 148 astrocytoma tissues (grades I-III) and 226 grade IV astrocytomas were quantified for expression levels.

cytoma, as compared to grade I. However, overexpressing and knocking down StarD13 led to a decrease and increase in cell proliferation, respectively, as would be expected of a tumor suppressor. In order to reconcile our results, we mined the REMBRANDT (Repository of Molecular Brain Neoplasia Data) database which hosts diverse types of molecular research and clinical trials data related to brain cancers, including gliomas. The results showed StarD13 is underexpressed in tumor tissues as compared to non-tumor tissues. However, if we compare the expression level in GBM (glioblastoma) which is grade IV astrocytoma to lower grade tumors, we find that the levels of StarD13 mRNA in grade IV is higher (Fig. 5). This was consistent with our IHC results.
StarD13 does not affect the apoptosis pathway. The effect of StarD13 on cell viability could be through inducing apoptosis. To study the effect of StarD13 on apoptosis, cells were stained with Annexin V-FITC. The fluorescence was immediately measured by a flow cytometry. Cells, which are early in the apoptotic process, will stain with the Annexin V-FITC conjugate alone. Live cells will show no staining by either the propidium iodide solution or Annexin V-FITC conjugate. Necrotic cells will be stained by both the propidium iodide solution and Annexin V-FITC conjugate. Our results showed that the knockdown of StarD13 did not affect apoptosis. The percentage of apoptotic cells was not significantly different between the controls and the transfected cells (Fig. 6 and Table I). 

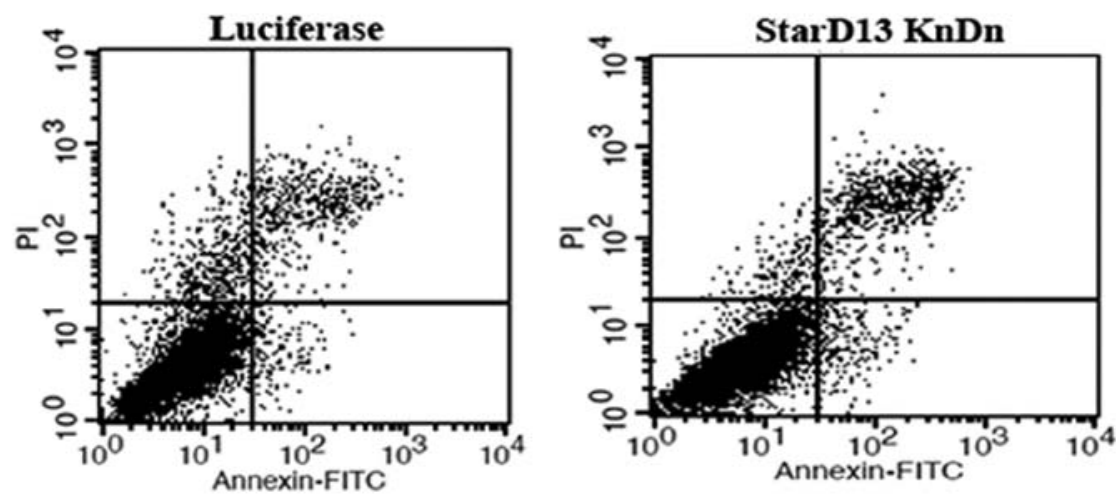

Figure 6. StarD13 had no significant effect on apoptosis. Cells were transfected with either control luciferase siRNA or with StarD13 siRNA. The cells were then trypsinized and stained with $5 \mu \mathrm{l}$ of Annexin V-FITC and $10 \mu \mathrm{l}$ of propidium iodide. The fluorescence of the cells was determined immediately with a flow cytometer. Cells, which are early in the apoptotic process, will stain with the Annexin V-FITC conjugate alone. Live cells will show no staining by either the propidium iodide solution or Annexin V-FITC conjugate. Necrotic cells will be stained by both the propidium iodide solution and Annexin V-FITC conjugate.
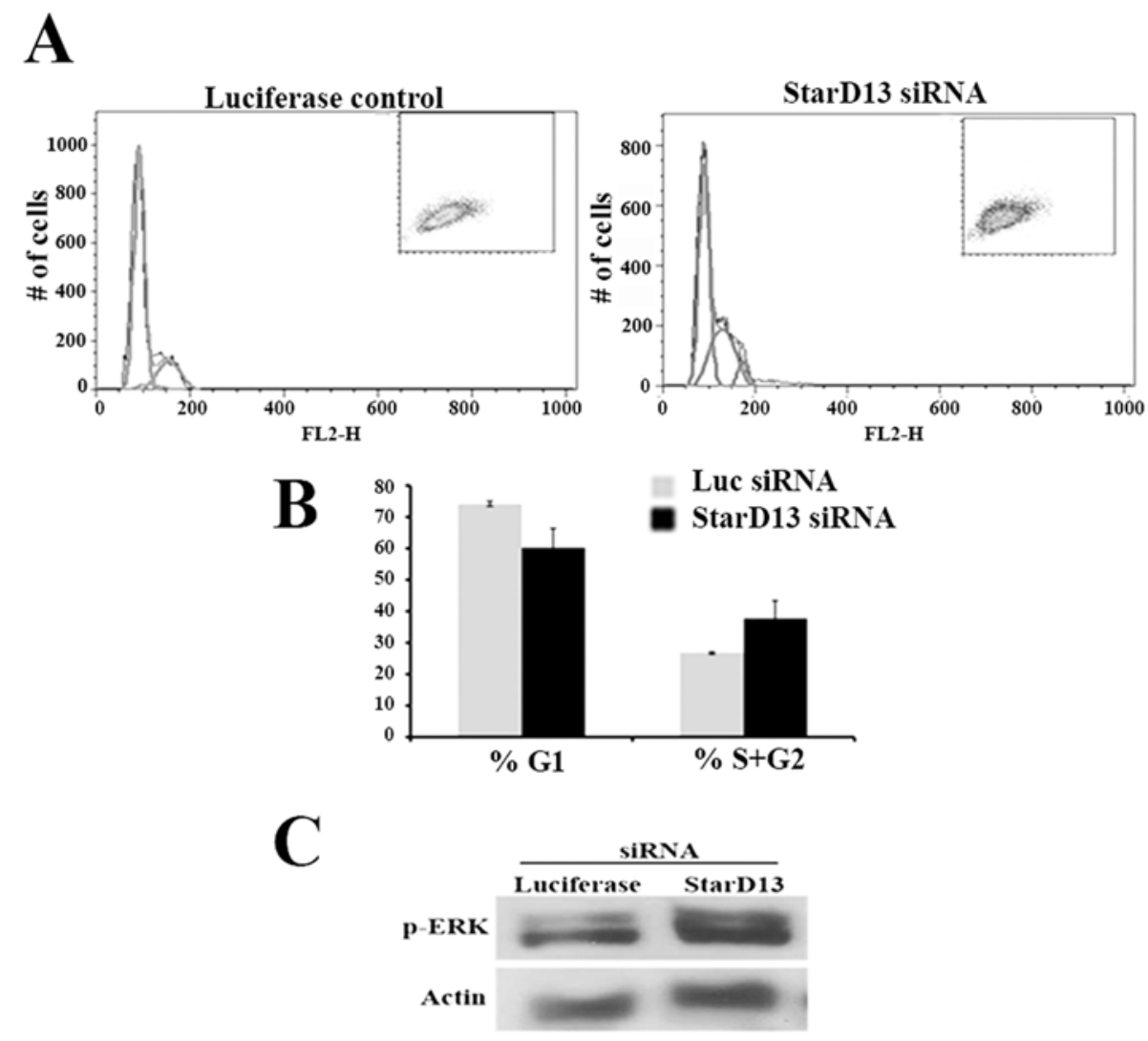

Figure 7. StarD13 represses the cell cycle. (A and B) Cells were transfected with luciferase or StarD13 siRNA and stained with $30 \mu 1$ propidium iodide for 10 min. Cells were analyzed using a FACScan, which indicated the distribution of the cells into their respective cell cycle phases based on their DNA content. G0/G1 cells were 2n; S-phase cells were $>2 \mathrm{n}$ but $<4 \mathrm{n}$ while G2/M were 4n. Cell DNA content was determined by CellQuest software. (C) StarD13 affects the cell cycle through an effect on ERK. Cells were transfected with luciferase (left lane) or StarD13 (right lane) siRNA, lyzed and blotted for p-ERK (upper gel) or actin for loading control (lower gel).

StarD13 affects the cell cycle. In order to understand the mechanism through which StarD13 is affecting cell viability, we analyzed the effect of StarD13 knockdown on the cell cycle. This was achieved using flow cytometry. Our results showed an increase in the percentage of cells in the $\mathrm{S} / \mathrm{G} 2$ phase as compared to the cells in the G0 phase (Fig. 7A and B). This showed that knocking down StarD13 led to an increase in cycling cells, meaning StarD13 plays the role of a tumor suppressor inhibiting the cell cycle.

ERK is a downstream effector of StarD13. In order to determine the mechanism of action of StarD13, we knocked it down and looked at the effect on p-ERK, the extracellular signal-regulated kinase. The western blotting showed an increase in pERK levels 
Table I. StarD13 had no significant effect on apoptosis.

\begin{tabular}{lcc}
\hline & Luciferase control & StarD13 KnDn \\
\hline Dead cells & 2.265 & 1.23 \\
Necrotic cells & 3.58 & 5.72 \\
Apoptotic cells & 1.21 & 2.42 \\
Live cells & 92.945 & 90.63 \\
\hline
\end{tabular}

Summary of the results in Fig. 6. Cells were transfected with either control luciferase siRNA or with StarD13 siRNA. The cells were then trypsinized and stained with $5 \mu \mathrm{l}$ of Annexin V-FITC and $10 \mu \mathrm{l}$ of propidium iodide. The fluorescence of the cells was determined immediately with a flow cytometer.

in siRNA-StarD13 transfected cells as compared to control non transfected cells (Fig. 7C).

\section{Discussion}

In this study, we examined the role of StarD13 in astrocytoma malignancy. We confirmed that StarD13 is a specific GAP for Rho and Cdc42. IHC analysis showed that StarD13 is overexpressed in grade IV astrocytoma compared to grade I. Mining online databases explained this observation by StarD13 being indeed overexpressed in grade IV astrocytoma as compared to grade I, however StarD13 is underexpressed in astrocytoma (grades I and IV) as compared to normal tissues. This confirmed StarD13 as a potential tumor suppressor in astrocytoma. In order to directly prove that, we overexpressed or knocked down StarD13 and looked at the effect of cell viability, apoptosis and proliferation in a cell culture model. In astrocytoma cell lines, overexpressing StarD13 led to no effect on apoptosis but to a decrease in cell viability and cell proliferation as reflected by a decrease in cells in S and G2 phase. Knocking down StarD13 with StarD13 siRNA led to no effect on cell apoptosis but to an increase in cell viability and cell proliferation. Knocking down StarD13 also showed an increase in phosphorylated ERK.

Ching et al was the first to identify and characterize StarD13 in hepatocellular carcinoma (HCC) (21). We wanted to determine the role of this RhoGAP in another tumor model which is astrocytoma. Ching et al reported the function of StarD13 as a GAP for RhoA and Cdc42 not for Rac1 (21). Our results demonstrated that cells overexpressing StarD13 possessed reduced levels of RhoA and Cdc42 activation; however, the activation of Racl was not affected. Therefore, similar to its role in HCC, StarD13 has a RhoGAP activity for RhoA and $\mathrm{Cdc} 42$ in astrocytoma. The IHC analysis on grades I-IV brain tissues from patients showed StarD13 to be overexpressed in grades III and IV astrocytoma tumors when compared to grades I and II. This was contradictory to other studies where the StarD13 gene was found to be downregulated in several types of cancer including lung, ovarian, renal, breast, uterine, gastric, colon and rectal tumors (23). These results led us to formulate the following hypothesis: contrary to other tumor models, in brain astrocytomas, StarD13 seems to be an oncogene and not a tumor suppressor. The REMBRANDT data, however, showed that the mRNA levels of StarD13 are indeed higher in the higher grades but much lower than the normal tissues. Hence, our IHC results were consistent with role of StarD13 as a tumor suppressor in astrocytoma, similar to hepatocellular carcinoma. We also showed that StarD13 overexpression leads a decrease in the number of viable cells, proving directly that StarD13 is a tumor suppressor.

It could be informative to establish the significance of the overexpression of StarD13 as the malignancy of astrocytoma increases. A present study in our laboratory showed that StarD13 is needed for astrocytoma cell motility. This might explain why StarD13 is overexpressed in grade IV astrocytoma compared to grade I. This pattern was also observed in the case of DLC1, which although a known tumor suppressor, was found to be needed for the motility of normal prostate cells (26). Could a protein play different or even opposing roles during the course of carcinogenesis? This remains to be investigated.

The effect of cell viability could be either due to decreased proliferation or increased apoptosis. To test which underlying mechanism was responsible for the tumor suppressor function of StarD13, we studied the effect of StarD13 knockdown on cell cycle using flow cytometry. In StarD13 siRNA transfected cells, the percentage of cells at the $\mathrm{S} / \mathrm{G} 2$ phase was higher than that of the control cells. The percentage of cells at the G1 phase was lower. These results indicated that the deleted in liver cancer 2 blocks the cells in the G1 phase inhibiting the cell cycle progression. This was consistent with a study conducted by Leung et al (27) which demonstrated that a stable expression of StarD13 caused accumulation of cells in the G1 phase leading to the inhibition of cell growth.

In addition to the RhoGAP domain, the START domain plays an important role by targeting the tumor suppressor DLC2 to mitochondria, indicating a possible role of DLC2 in lipid transport and in the regulation of the mitochondrial pathway of apoptosis and mitochondrial membrane permeability (22). Therefore, we examined the effect of StarD13 on apoptosis. Our results showed that StarD13 did not induce apoptosis. This was reflected by the percentage of apoptotic cells which was approximately similar in the StarD13 siRNA transfected cells and the control cells.

To examine the underlying mechanism behind the tumor suppressor role of StarD13, we investigated the level of ERK phosphorylation after the silencing of StarD13. The results showed an increase in the level of p-ERK in the cells transfected with StarD13 siRNA as compared to non-transfected cells. These findings were in accordance with the study conducted by Leung et al which showed that StarD13 suppresses cell growth via the regulation of the Raf1-ERK1/2-p70S6K signaling pathway (27). Since p-ERK, the extracellular signal-regulated kinase, is involved in the regulation of cellular growth and proliferation of several tumor types (28), we suggest that possibly our StarD13 is affecting the cell growth of tumor cells via p-ERK pathway. This is of interest since it directly links Rho or Cdc42 to the inhibition of a MAPK pathway.

This study confirmed the role of StarD13 as a tumor suppressor in astrocytoma. It also uncovered a link between the Rho GTPase pathway and the MAPK pathway. Perhaps the most interesting finding in this study was the variation in the level of expression of a tumor suppressor based on the grade of the tumor. This corroborated the fact that the same protein might play different and even opposing roles in the cell depending on the conditions. 


\section{Acknowledgements}

The authors would like to thank Dr Noha Bajjani, from Rizk Medical Center for the tissues collected. We would also like to thank Dr Hitoshi Yagisawa for providing constructs. This study was supported by the Natural Science Department at the Lebanese American University, by the University Research Council (URC) at LAU and by the Lebanese National Center for Scientific Research (L-NCSR) (Ref: 03-06-10).

\section{References}

1. Buckner JC, Brown PD, O'Neill BP, Meyer FB, Wetmore CJ and Uhm JH: Central nervous system tumors. Mayo Clin Proc 82: 1271-1286, 2007.

2. Salacz ME, Watson KR and Schomas DA: Glioblastoma: Part I. Current state of affairs. Mol Med 108: 187-194, 2011.

3. Salacz ME, Watson KR and Schomas DA: Glioblastoma. Part II: future directions. Mol Med 108: 289-291, 2011.

4. Louis DN, Ohgaki H, Wiestler OD and Cavenee WK: WHO Classification of Tumours of the Central Nervous System. World Health Organization, 2007.

5. Wen PY and Kesari S: Malignant gliomas in adults. N Engl J Med 359: 492-507, 2008.

6. DeAngelis LM: Brain tumors. N Engl J Med 344: 114-123, 2001.

7. Karlsson R, Pedersen ED, Wang Z and Brakebusch C: Rho GTPase function in tumorigenesis. Biochim Biophys Acta 1796: 91-98, 2009.

8. Boettner B and Van Aelst L: The role of Rho GTPases in disease development. Gene 286: 155-174, 2002.

9. Ridley AJ: Rho proteins and cancer. Breast Cancer Res Treat 84: 13-19, 2004.

10. Hornstein I, Pikarsky E, Groysman M, Amir G, Peylan-Ramu N and Katzav S: The haematopoietic specific signal transducer Vav1 is expressed in a subset of human neuroblastomas. J Pathol 199: 526-533, 2003

11. Wang W, Wyckoff JB, Frohlich VC, et al: Single cell behavior in metastatic primary mammary tumors correlated with gene expression patterns revealed by molecular profiling. Cancer Res 62: 6278-6288, 2002.

12. Kim TY, Vigil D, Der CJ and Juliano RL: Role of DLC-1, a tumor suppressor protein with RhoGAP activity, in regulation of the cytoskeleton and cell motility. Cancer Metastasis Rev 28: 77-83, 2009.

13. Kawai K, Seike J, Iino T, et al: START-GAP2/DLC2 is localized in focal adhesions via its N-terminal region. Biochem Biophys Res Commun 380: 736-741, 2009.
14. Rittinger K, Walker PA, Eccleston JF, et al: Crystal structure of a small $G$ protein in complex with the GTPase-activating protein rhoGAP. Nature 388: 693-697, 1997.

15. Yau TO, Leung TH, Lam S, et al: Deleted in liver cancer 2 (DLC2) was dispensable for development and its deficiency did not aggravate hepatocarcinogenesis. PLoS One 4: e6566, 2009.

16. Tcherkezian J and Lamarche-Vane N: Current knowledge of the large RhoGAP family of proteins. Biol Cell 99: 67-86, 2007.

17. Soccio RE and Breslow JL: StAR-related lipid transfer (START) proteins: mediators of intracellular lipid metabolism. J Biol Chem 278: 22183-22186, 2003.

18. Qian X, Li G, Asmussen HK, et al: Oncogenic inhibition by a deleted in liver cancer gene requires cooperation between tensin binding and Rho-specific GTPase-activating protein activities. Proc Natl Acad Sci USA 104: 9012-9017, 2007.

19. Li H, Fung KL, Jin DY, et al: Solution structures, dynamics, and lipid-binding of the sterile alpha-motif domain of the deleted in liver cancer 2. Proteins 67: 1154-1166, 2007.

20. Liao YC and Lo SH: Deleted in liver cancer-1 (DLC-1): a tumor suppressor not just for liver. Int J Biochem Cell Biol 40: 843-847, 2008.

21. Ching YP, Wong CM, Chan SF, et al: Deleted in liver cancer (DLC) 2 encodes a RhoGAP protein with growth suppressor function and is underexpressed in hepatocellular carcinoma. $\mathrm{J}$ Biol Chem 278: 10824-10830, 2003.

22. Ng DC, Chan SF, Kok KH, et al: Mitochondrial targeting of growth suppressor protein DLC2 through the START domain. FEBS Lett 580: 191-198, 2006.

23. Ullmannova $\mathrm{V}$ and Popescu NC: Expression profile of the tumor suppressor genes DLC-1 and DLC-2 in solid tumors. Int J Oncol 29: 1127-1132, 2006

24. Leung TH, Ching YP, Yam JW, et al: Deleted in liver cancer 2 (DLC2) suppresses cell transformation by means of inhibition of RhoA activity. Proc Natl Acad Sci USA 102: 15207-15212, 2005.

25. Xiaorong L, Wei W, Liyuan Q and Kaiyan Y: Underexpression of deleted in liver cancer 2 (DLC2) is associated with overexpression of RhoA and poor prognosis in hepatocellular carcinoma. BMC Cancer 8: 205, 2008.

26. Shih YP, Takada Y and Lo SH: Silencing of DLC1 upregulates PAI-1 expression and reduces migration in normal prostate cells. Mol Cancer Res 10:34-39, 2012.

27. Leung LH, Wong WK, Cheng AC, et al: A new approach to computing normal tissue complication probability of an intensitymodulated radiotherapy treatment with stereotactic radiotherapy boost of nasopharyngeal carcinoma: a case study. Med Dosim 36: 138-144, 2011.

28. Zheng B, Fiumara P, Li YV, et al: MEK/ERK pathway is aberrantly active in Hodgkin disease: a signaling pathway shared by CD30, CD40, and RANK that regulates cell proliferation and survival. Blood 102: 1019-1027, 2003. 